\title{
COMPÓSITOS POLIMÉRICOS DE MATRIZ DE POLIPROPILENO E TERMOPLÁSTICO DE AMIDO COM BIOMASSA FÚNGICA DE ASPERGILLUS SP E PENICILLIUM SP
}

\author{
Bruno Felipe Bergel ${ }^{1}$ \\ Leonardo Azevedo Massulo ${ }^{2}$ \\ Valeriano Antonio Corbellini ${ }^{3}$ \\ Adriane de Assis Lawisch Rodriguez ${ }^{4}$ \\ Claudia Mendes Mahlmann ${ }^{5}$
}

\begin{abstract}
RESUMO
Os materiais biodegradáveis, ou parcialmente degradáveis, tem ganhado espaço nos últimos tempos devido ao aumento das políticas que visam controlar e diminuir os problemas ambientais. Dentro deste cenário, este trabalho teve o objetivo de estudar compósitos poliméricos parcialmente biodegradáveis, utilizando termoplástico de amido (TPS) e biomassa fúngica provinda dos gêneros Aspergillus sp e Penicillium $s p$. Para a confecção destes compósitos, utilizou-se como matriz uma blenda com $20 \%$ de TPS e $80 \%$ de polipropileno (PP) em massa. Como fase dispersa foi utilizada biomassa fúngica em fração mássica de 5\%. O principal objetivo da adição desta biomassa foi a possibilidade de aumento das propriedades mecânicas, sem alterar as características biodegradáveis do material. Os compósitos foram cortados em corpos de prova e estes foram avaliados através de ensaios de tração, flexão e dureza. Os resultados indicaram a dependência das propriedades das amostras, considerando o tipo de fase dispersa na formulação e também a possibilidade de uso deste material para diversas aplicações.
\end{abstract}

Palavras-chave: Compósito. Blenda. TPS. Aspergillus sp. Penicillium sp.

\begin{abstract}
Biodegradable materials, or partially degradable, have gained space in recent times due to the increase of policies to control and reduce environmental problems. Within this scenario, the objective of this work was to study partially biodegradable polymer composites using thermoplastic starch (TPS) and fungal biomass of the genus Aspergillus sp. and Penicillium $s p$. To prepare these composites was used as matrix a blend with $20 \%$ TPS and $80 \%$ polypropylene (PP) by mass. Fungal biomass was used as the dispersed phase in mass fraction of 5\%. The main purpose of adding this biomass was the possibility of increased mechanical properties without changing the characteristics of biodegradable material. The composites were cut into test bodies and these were evaluated by tensile, bending and hardness. The results indicate the dependence of the properties of the samples considering the type of

\footnotetext{
1 Aluno do Curso de Química Industrial da Universidade de Santa Cruz do Sul - UNISC. < brunobergel@hotmail.com>

2 Aluno do Curso de Engenharia Civil da Universidade de Santa Cruz do Sul - UNISC. <leonardomassulo@yahoo.com.br>

3 Professor do Departamento de Química e Física na Universidade de Santa Cruz do Sul - UNISC. <valer@unisc.br>

4 Professora do Departamento de Engenharia, Arquitetura e Ciências Agrárias na Universidade de Santa Cruz do Sul - UNISC. <adriane@unisc.br>

5 Professora do Departamento de Química e Física na Universidade de Santa Cruz do Sul - UNISC. <mclaudia@unisc.br>
} 
formulation in the dispersed phase and the possibility of using this material for many applications.

Keywords: Composite. Blend. TPS. Aspergillus sp. Penicillium sp.

\section{INTRODUÇÃO}

Por conta da demanda por novos materiais que não agridam o meio ambiente, os materiais biodegradáveis tornaram-se de suma importância. Dessa forma, as políticas de sustentabilidade têm investido cada vez mais na procura de materiais com propriedades interessantes e que não poluam o ambiente. Neste contexto está o termoplástico de amido (TPS), que puro, possui propriedades mecânicas ruins, mas quando misturado com poliolefinas forma blendas que podem apresentar propriedades interessantes. Este tipo de blenda pode ser utilizado também como matriz para compósitos, com o objetivo de incrementar suas propriedades mecânicas e de manter sua biodegradabilidade. Neste sentido, pode-se adicionar biomassa fúngica ou fibras naturais como fase dispersa.

Este trabalho teve como objetivo a elaboração e formação de compósitos com termoplástico de amido e polipropileno como matriz, biomassa fúngica de Aspergillus sp. e de Penicillium sp. e compósitos de cana-de-açúcar como fase dispersa. Estes compósitos foram avaliados de acordo com suas propriedades em tração, flexão e dureza.

\section{FUNDAMENTAÇÃO TEÓRICA}

\subsection{Biomassa Fúngica de Aspergillus sp., Penicillium sp. e Fibra de Cana-de-Açúcar}

Fibras naturais tem sido apontadas como soluções para a produção de compósitos, pois podem atuar como reforço nestes. Além disso, possuem baixo custo e grande compatibilidade com o meio ambiente. Dentre as fibras que podem ser utilizadas estão as fibras de cana-de açúcar, que são um resíduo da agroindústria brasileira, e as fibras de biomassa fúngica. (SANCHEZ, 2010)

Os gêneros de fungos Aspergillus sp. e Penicillium $s p$. estão presentes em praticamente todos os cantos do mundo, crescendo facilmente nos mais diversos materiais, como queijos e frutas. Pelo fato de serem gêneros comuns e fáceis de encontrar, aliados ao fácil crescimento da biomassa, tornam-se ideais para a produção de biomassa. Os emaranhados de hifas destes fungos formam um micélio forte, o que pode ajudar em propriedades físicas em conjunto com outros materiais (HEO, 2013). Já a fibra de cana-de- 
açúcar é um resíduo da produção de cana-de-açúcar e também pode ajudar na fortificação de propriedades físicas e mecânicas de compósitos, pois estas fibras são longas e resistentes. Elas também tendem a ter boas propriedades de isolamento térmico, elétrico e acústico (SANCHEZ, 2010).

\subsection{Termoplástico de Amido de Mandioca}

O termoplástico de amido é um polímero biodegradável a base de amido. Para a produção deste plástico é necessário juntar ao amido um plastificante, geralmente glicerol. Este plastificante serve para melhorar a processabilidade e aumentar a flexibilidade do polímero (TEIXEIRA et al; 2012).

Este plástico biodegradável torna-se interessante porque existem várias fontes renováveis abundantes de amido, e este se torna fácil de conseguir. Porém, em contrapartida às suas vantagens, existem algumas dificuldades. Uma delas é que o material é muito sensível ao contato da água e tende a se diluir nela. Outro problema é que este termoplástico puro tende a possuir propriedades mecânicas fracas. Uma maneira de resolver ou minimizar estes problemas é juntar este plástico a outros, por meio de blendagem (SCHLEMMER, 2007).

\subsection{Blendagem}

O processo de blendagem consiste em misturas físicas de polímeros com estruturas diferentes que interagem juntos através de ligações de força secundária. A miscibilidade constitui a propriedade mais importante em blendas poliméricas. Há blendas miscíveis e imiscíveis, e isto dependerá dos tipos de materiais empregados e suas respectivas solubilidades um no outro (BODOR, 1991).

Blendas produzidas a partir de poliolefinas (como o polipropileno) e polímeros biodegradáveis costumam apresentar biodegradabilidade elevada, principalmente devido ao contato de vários grupos hidroxilas (RAMIS et al. 2004). Por outro lado, geralmente apresentam problemas de miscibilidade, uma vez que um é hidrofóbico e, o outro, hidrofílico.

\subsection{Compósitos}

Compósitos poliméricos são materiais multifásicos que apresentam uma relevância significativa das propriedades das partes que o constituem. O principal objetivo destes compósitos é formar novos materiais a partir de componentes unitários, e que estes novos materiais apresentem propriedades superiores ao dos componentes sozinhos, ou que pelo 
menos algumas propriedades sejam melhoradas em relação a um dos componentes, dependendo da aplicação que este material terá. Geralmente a matriz polímerica é um polímero ou uma blenda que irá formar a base do compósito. Já a fase dispersa irá dar propriedades específicas ao compósito, assim como elevar outras (ALLCOCK, 1990). Nos últimos anos, a utilização de fibras naturais como fase dispersa cresceu muito, pois estas se adaptam bem ao polímero (considerando a biodegradabilidade deste tipo de material e são oriundos de fonte renovável) e possuem baixo custo e alta biodegradabilidade (YU et al. 2006).

\section{MATERIAIS E MÉTODOS}

\subsection{Materiais e Equipamentos}

Foram utilizados no desenvolvimento deste trabalho: polipropileno (PP) EP448RQUATTOR, amido de mandioca e glicerol Synth 99,5\% de pureza.

Os equipamentos utilizados na realização deste trabalho foram: forno de micro-ondas convencional (Panasonic, potência de $2450 \mathrm{MHz}, 1100 \mathrm{~W} / 1600 \mathrm{~W}$ ); moinho de facas Mecanofar - MF300; extrusora monorosca SEIBT; homogeneizador de Laboratório MH-100 (MH Equipamentos); prensa hidráulica (MH Equipamentos); balança analítica (Quimis); estufa De Leo; microretífica (DREMEL); máquina Universal de Ensaios Mecânicos (EMIC DL 10.000) e acessórios, durômetro (Teclock Politest). Para a produção de biomassa foi utilizada a autoclave Phenix Equipment Scientific.

\subsection{Produção de Termoplástico de Amido (TPS)}

O termoplástico de amido (TPS) foi produzido a partir de amido de mandioca, glicerol e água em uma proporção de 16:4:80 (m/m/m). Após a mistura, levou-se para o forno micro-ondas em potência média por cerca de 7 minutos, com intervalos de 1 minuto para homogeneização. Feito isso, o termoplástico gelatinizou e foi posto em formas para ser seco em estufa e, sendo depois moído e armazenado em local longe de umidade.

\subsection{Produção de Biomassa Fúngica de Aspergillus sp. e Penicillium sp.}

As biomassas fúngicas de Aspergillus sp. e Penicillium sp. foram obtidas por meio do cultivo destes fungos em placas de petry e em erlenmeyers. Em um primeiro momento, as placas e os erlenmeyers foram levados para autoclave, por um período de 15 minutos, com o 
objetivo de esterilizar estes materiais. Após esta etapa, preparou-se um meio de cultivo nutritivo preparado a partir de glicose, peptona e água em uma proporção de 4:2:100 $(\mathrm{m} / \mathrm{m} / \mathrm{m})$. Estes ingredientes foram misturados e levados ao forno micro-ondas para uma completa homogeneização e, então, o meio nutritivo foi vertido para as placas e para os erlenmeyers. Em seguida, os fungos requeridos foram inoculados nos materiais e estes foram deixados em repouso por um período de 5 dias a uma temperatura de $30^{\circ} \mathrm{C}$, com o propósito de o fungo se ambientar e crescer, consumindo o meio nutritivo e desenvolvendo a biomassa fúngica. Após este período, a biomassa foi coletada, armazenada e submetida à esterilização em autoclave por um período de 1 hora. Então, esta biomassa foi seca em estufa e utilizada na fase dispersa do compósito.

As fibras de cana-de-açúcar, antes de serem utilizadas, foram lavadas em água e secas em estufa a $70^{\circ} \mathrm{C}$. Depois, foram moídas em moinho de facas e as fibras foram separadas pela sua granulometria, com o auxilio de peneiras. Para a formação dos compósitos, só foram utilizados as fibras com granulometria menor que $1 \mathrm{~mm}$.

\subsection{Produção dos Compósitos}

Os compósitos foram produzidos a partir de uma matriz de 8:2 (m/m) de PP/TPS, utilizando-se $5 \%$ de biomassa fúngica como fase dispersa. Este material foi extrusado com temperatura máxima de $165^{\circ} \mathrm{C}$, moído em moinho de facas e processado por moldagem por compressão. O material obtido foi uma placa de $2 \mathrm{~mm}$ de espessura e, desta forma, foram cortados os corpos de prova. Os resultados foram comparados a outros compósitos feitos em trabalhos anteriores.

\subsection{Caracterização das Propriedades}

Para analisar as propriedades das amostras processadas, foram realizados ensaios descritos como segue:

- Tração: foi realizado seguindo procedimento descrito na norma ASTM D638. Os ensaios foram realizados na máquina universal de ensaio EMIC DL 10.000, com velocidade do ensaio de $5 \mathrm{~mm} \mathrm{~min}^{-1}$.

- Flexão: foi realizado seguindo procedimento descrito na norma ASTM D790, na máquina universal de ensaio EMIC DL 10.000, com velocidade do ensaio de $5 \mathrm{~mm} \mathrm{~min}^{-1}$. 
- Determinação de dureza Shore D: foi realizado conforme procedimento descrito na norma ASTM-D2240, com tempo de medida de 3s no equipamento Durômetro Shore GSD 702 Teclock Politest, realizado em triplicata.

\section{RESULTADOS E DISCUSSÕES}

\subsection{Ensaio de Tração}

Os resultados obtidos através de ensaio de tração para o parâmetro módulo de elasticidade da blenda polimérica de PP + TPS 20\%, dos compósitos de PP/TPS + 5\% de biomassa de Aspergillus sp., de PP/TPS + 5\% de biomassa de Penicillium sp. e de PP/TPS + 5\% de fibra de cana, além dos compósitos sem TPS, são apresentados na Fig. 1.

\section{Figura 1 - Resultados do Ensaio de Tração para o parâmetro módulo de elasticidade}

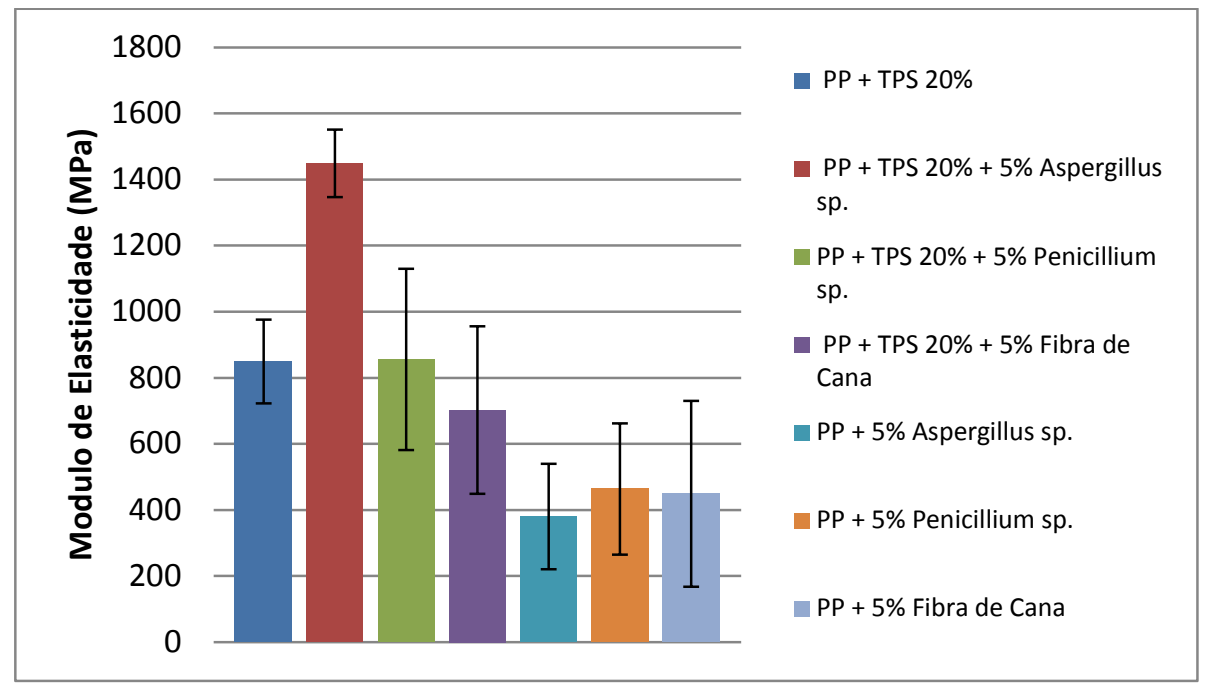

Foi observado o aumento de rigidez no compósito contendo 5\% de Aspergillus sp. em relação ao PP/TPS puro. Já a adição de 5\% de fibra de cana ao PP/TPS tornou este compósito menos rígido e mais flexível comparado aos outros materiais. Os compósitos sem TPS mostraram-se bem menos rígidos que os demais.

A Fig. 2 apresenta os resultados de tensão máxima no ensaio de tração. 
Figura 2 - Resultado do Ensaio de Tração para o parâmetro Tensão na força máxima

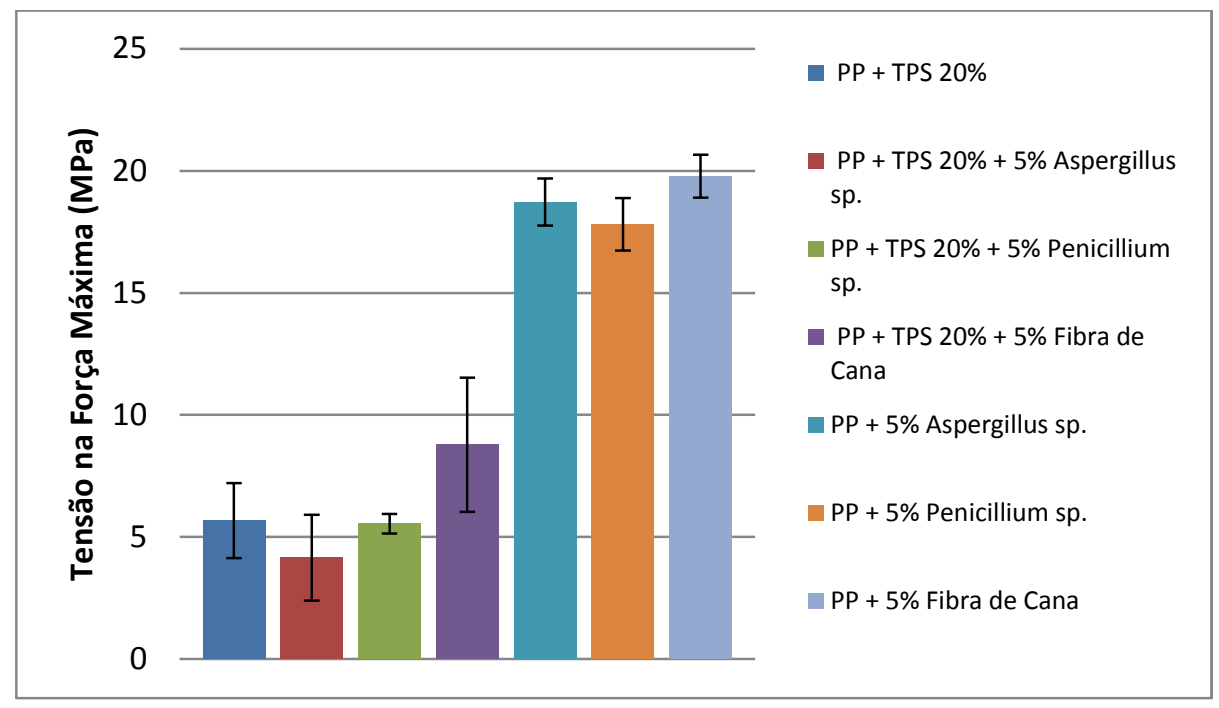

Verificou-se um grande aumento de resistência mecânica nos compósitos sem a presença de TPS. Dentre os compósitos com a presença de TPS, o que apresentou um melhor resultado foi o com fibra de cana. Nos compósitos sem a presença de TPS, a tendência continuou a mesma, e o compósito de fibra de cana mostrou-se mais resistente.

\subsection{Ensaio de Flexão}

Os valores obtidos para o módulo de elasticidade do ensaio de flexão das amostras testadas são apresentados na Fig. 3.

\section{Figura 3 - Resultado do parâmetro módulo de elasticidade no ensaio de flexão}

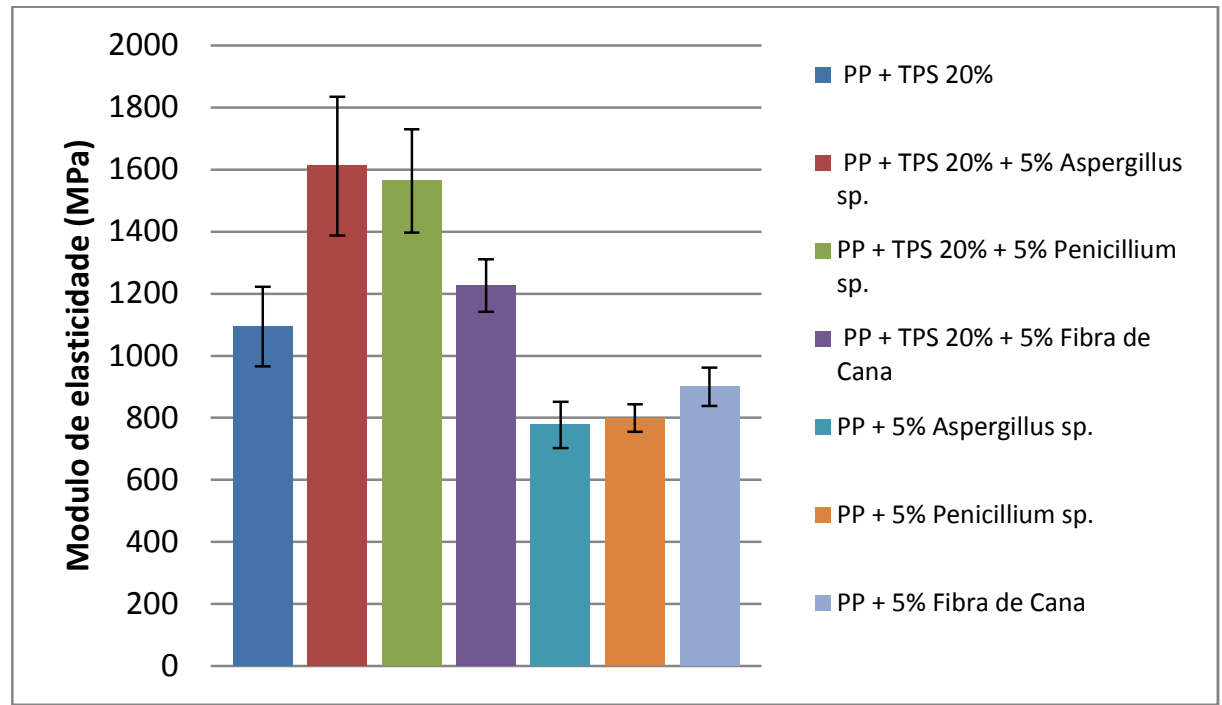


Os resultados mostraram que os compósitos de 5\% Aspergillus sp e de 5\% Penicillium $s p$. possuem grande rigidez em comparação com os outros materiais. O compósito de $5 \%$ fibra de cana apresenta-se mais flexível do que os de biomassa, possivelmente devido ao formato das fibras de cana, que facilita a flexão. Já a adição das biomassas fúngicas fez com que o material ficasse mais rígido, assim como no ensaio de tração. Os compósitos sem TPS mostraram-se novamente menos rígidos que os demais.

O resultado da tensão máxima no ensaio de flexão é apresentado na Fig. 4.

\section{Figura 4 - Resultados de Tensão Máxima no ensaio de flexão}

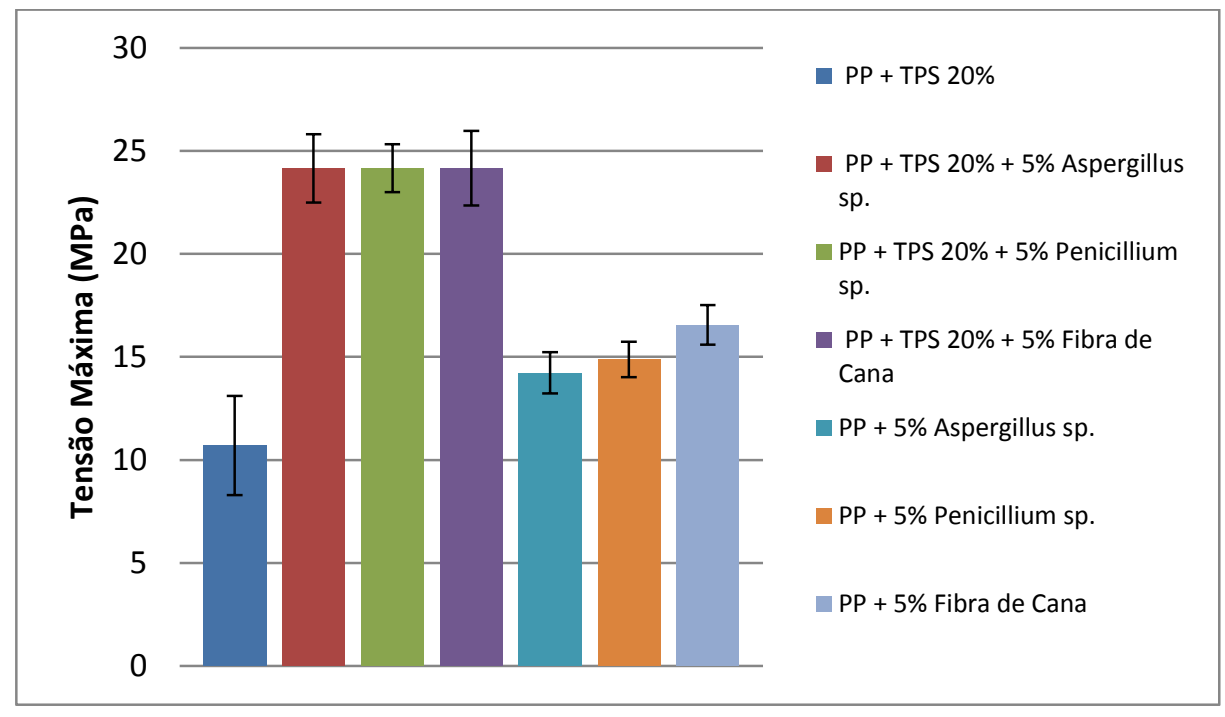

Verificou-se que os compósitos com 5\% de fibra de cana, 5\% de Penicillium sp. e com 5\% de Aspergillus sp apresentaram resistência mecânica em flexão parecida, e ambos exibiram uma resistência melhor do que o PP/TPS puro. Já os compósitos sem TPS apresentaram resistência inferior aos com TPS, mas superior ao PP/TPS puro. 


\subsection{Ensaio de Dureza}

O resultado do ensaio de dureza é apresentado na Fig. 5.

Figura 5 - Resultados do ensaio de dureza (Shore D)

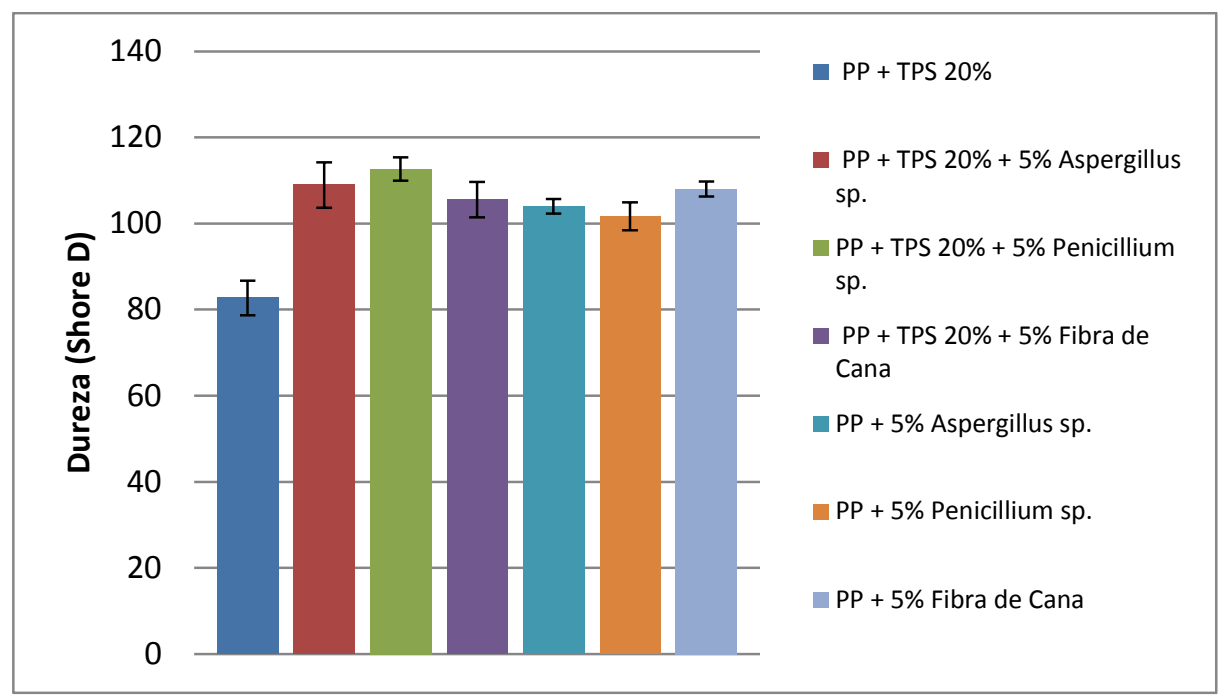

Observou-se uma maior dureza superficial dos compósitos em relação ao PP/TPS puro. Nos compósitos contendo TPS, os compósitos de Aspergillus sp.e de Penicillium sp. mostraram-se mais duros em comparação com o de cana-de-açúcar. Já nos compósitos sem a presença de TPS, o compósito de cana-de-açúcar apresentou uma dureza mais elevada em comparação ao demais.

\section{CONCLUSÃO}

A partir dos parâmetros avaliados e dos resultados obtidos, verificou-se que a adição de biomassas de Aspergillus sp. e de Penicillium sp. melhoraram propriedades do material em comparação a matriz PP/TPS puro, apresentando-se como um material mais rígido e duro, porém ocorreu perda de resistência mecânica em tração. Já os compósitos sem a presença de TPS mostraram melhorias na resistência mecânica em tração.

O compósito de fibra de cana também apresentou melhoria de propriedades em comparação com a matriz pura, mostrando-se mais flexível e menos duro.

No geral, os compósitos revelaram propriedades distintas e dependentes das suas respectivas fases dispersas, tendo sido verificada a função das mesmas como reforço na matriz estudada. 


\section{REFERÊNCIAS}

ALLCOCK, H. R.; LAMPE, F. W. Contemporary polymer chemistry. 2. ed. Englewood Cliffs: Prentice Hall, 1990.

BODOR, G. Structural investigation of polymers. New York: Ellis Horwood, 1991.

DUC, A. L.; VERGNES, B.; BUDTOVA, T. Polypropylene/natura fibres composites: analysis of fibre dimensions after compounding and observations of fibre rupture by rheooptics. Composites Part A: Applied Science and Manufacturing [online], n. 11, v. 42, p. 17271737, nov. 2011.

HEO, S.; JEON, S.; LEE, S. Utilization of Lentinus edodes mushroom $\beta$-glucan to enhance the functional properties of gluten-free rice noodles. Food Science and Technology [online], n. 2, v. 55, p. 627-631, mar. 2013.

ISHIZAKI, M. H.; FURTADO C. R. G.; LEBLANC J. L. Caracterização mecânica e morfológica de compósitos de polipropileno e fibras de coco verde: influência do teor de fibra e das condições de mistura. Polímeros: Ciência e Tecnologia, São Carlos, n. 3, v. 16, p. 182186, 2006.

MA, X.; YU, J.; KENNEDY, J. F. Studies on the properties of natural fibers-reinforced thermoplastic starch composites. Carbohydrates Polymers [online], n. 1, v. 62, p. 19-24, out. 2005.

RAMIS, X. et al. Thermal degradation of polypropylene/starch-based materials with enhanced biodegradability. Polymer Degradation and Stability [online], n. 3, v. 86, p. 483-491, dez. 2004.

RODRIGUEZ-GONZALEZ, F. J.; RAMSAY, B. A.; FAVIS, B. D. Rheological and thermal properties of thermoplastic starch with high glycerol content. Carbohydrate Polymers [online], n. 2, v. 58, p. 139-147, nov. 2004.

SANCHEZ, E. M. S. et al. Compósito de resina de poliéster insaturado com bagaço de canade-açúcar: influência do tratamento das fibras nas propriedades. Polímeros: Ciência $e$ Tecnologia, São Carlos, n. 3, v. 20, p. 194-200, jul./set. 2010.

SCHLEMMER, D. Preparação, caracterização e degradação de blendas de poliestireno e amido termoplástico usando glicerol e óleo de buriti (Mauritia flexuosa) como plastificantes. Brasília, 2007. Dissertação (Mestrado), Instituto de Química, Universidade de Brasília, 2007.

SOBCZAK, L. L. R. W.; HAIDER, A. A. Polypropylene composites with natural fibers and wood - general mechanical property profiles. Composites Science and Technology [online], n. 5, v. 72, p. 550-557, mar. 2012.

SPINACÉ, M. A. da S., DE PAOLI, M. A. A tecnologia da reciclagem de polímeros. Química Nova, São Paulo, n. 1, v. 28, p. 65-72, jan./fev. 2005. 
TEIXEIRA, E. D. M. et al. Properties of thermoplastic starch from cassava bagasse and cassava starch and their blends with poly (latic acid). Industrial Crops and Products [online], v. 37 , p. $61-68,2012$

YU, L.; DEAN, K.; LI, L. Polymer blends and composites from renewable resources. Process in Polymer Science [online], n. 6, v. 31, p. 576-602, jun. 2006. 